\title{
Corrigendum: Coronavirus Disease-2019 Survival in Mexico: A Cohort Study on the Interaction of the Associated Factors
}

\author{
Horacio Márquez-González ${ }^{1}$, Jorge F. Méndez-Galván ${ }^{2}$, Alfonso Reyes-López ${ }^{3}$, \\ Miguel Klünder-Klünder ${ }^{4}$, Rodolfo Jiménez-Juárez ${ }^{5}$, Juan Garduño-Espinosa ${ }^{6}$ and \\ Fortino Solórzano-Santos ${ }^{7 *}$ \\ ${ }^{1}$ Department of Clinical Research, Hospital Infantil de México Federico Gómez, Mexico City, Mexico, ${ }^{2}$ Centre for Research in \\ Emerging Diseases, Hospital Infantil de México Federico Gómez, Mexico City, Mexico, ${ }^{3}$ Centre for Health Economics, \\ Hospital Infantil de México Federico Gómez, Mexico City, Mexico, ${ }^{4}$ Research Management, Hospital Infantil de México \\ Federico Gómez, Mexico City, Mexico, ${ }^{5}$ Clinical Infectious Disease Department, Hospital Infantil de México Federico Gómez, \\ Mexico City, Mexico, ${ }^{6}$ Clinical Research Direction, Hospital Infantil de México Federico Gómez, Mexico City, Mexico, \\ 7 Infectious Diseases Research Department, Hospital Infantil de México Federico GómezMéxico Federico Gómez, Mexico \\ City, Mexico
}

Keywords: Mexico, SARS-CoV-2, survival analysis, cohort study, comorbidity, COVID-19 outbreak

\section{OPEN ACCESS}

Edited and reviewed by: Burc Barin,

Emmes Corporation, United States

${ }^{*}$ Correspondence: Fortino Solórzano-Santos solorzanof056@gmail.com

Specialty section

This article was submitted to Infectious Diseases - Surveillance, Prevention and Treatment,

a section of the journa

Frontiers in Public Health

Received: 06 August 2021

Accepted: 12 August 2021

Published: 29 September 2021

Citation:

Márquez-González $\mathrm{H}$, Méndez-Galván JF, Reyes-López A, Klünder-Klünder M, Jiménez-Juárez $R$,

Garduño-Espinosa J and

Solórzano-Santos F (2021)

Corrigendum: Coronavirus

Disease-2019 Survival in Mexico: A Cohort Study on the Interaction of the

Associated Factors.

Front. Public Health 9:754703. doi: 10.3389/fpubh.2021.754703

\section{A Corrigendum on}

Coronavirus Disease-2019 Survival in Mexico: A Cohort Study on the Interaction of the Associated Factors

by Márquez-González, H., Méndez-Galván, J. F., Reyes-López, A., Klünder-Klünder, M., JiménezJuárez, R., Garduño-Espinosa, J., and Solórzano-Santos, F. (2021). Front. Public Health 9:660114. doi: 10.3389/fpubh.2021.660114

In the original article, there were some errors. "Comorbidities" was misspelled. All instances of the incorrect spelling should be corrected to read "comorbidities".

The acronym for chronic obstructive pulmonary disease was incorrectly spelled. This should be COPD.

In the original articles, there were some mistakes in Table 2.

The section "Taquism" should be named "Smoking". The row "Taquism" in the "Outcomes" section was deleted. "Immunossupression" should read "Immunocompression".

The corrected table appears below.

The authors apologize for these errors and state that this does not change the scientific conclusions of the article in any way. The original article has been updated.

Publisher's Note: All claims expressed in this article are solely those of the authors and do not necessarily represent those of their affiliated organizations, or those of the publisher, the editors and the reviewers. Any product that may be evaluated in this article, or claim that may be made by its manufacturer, is not guaranteed or endorsed by the publisher.

Copyright ( 2021 Márquez-González, Méndez-Galván, Reyes-López, Klünder-Klünder, Jiménez-Juárez, Garduño-Espinosa and Solórzano-Santos. This is an open-access article distributed under the terms of the Creative Commons Attribution License (CC $B Y)$. The use, distribution or reproduction in other forums is permitted, provided the original author(s) and the copyright owner(s) are credited and that the original publication in this journal is cited, in accordance with accepted academic practice. No use, distribution or reproduction is permitted which does not comply with these terms. 
TABLE 2 | Differences between survivors and non-survivors with SARS CoV-2.

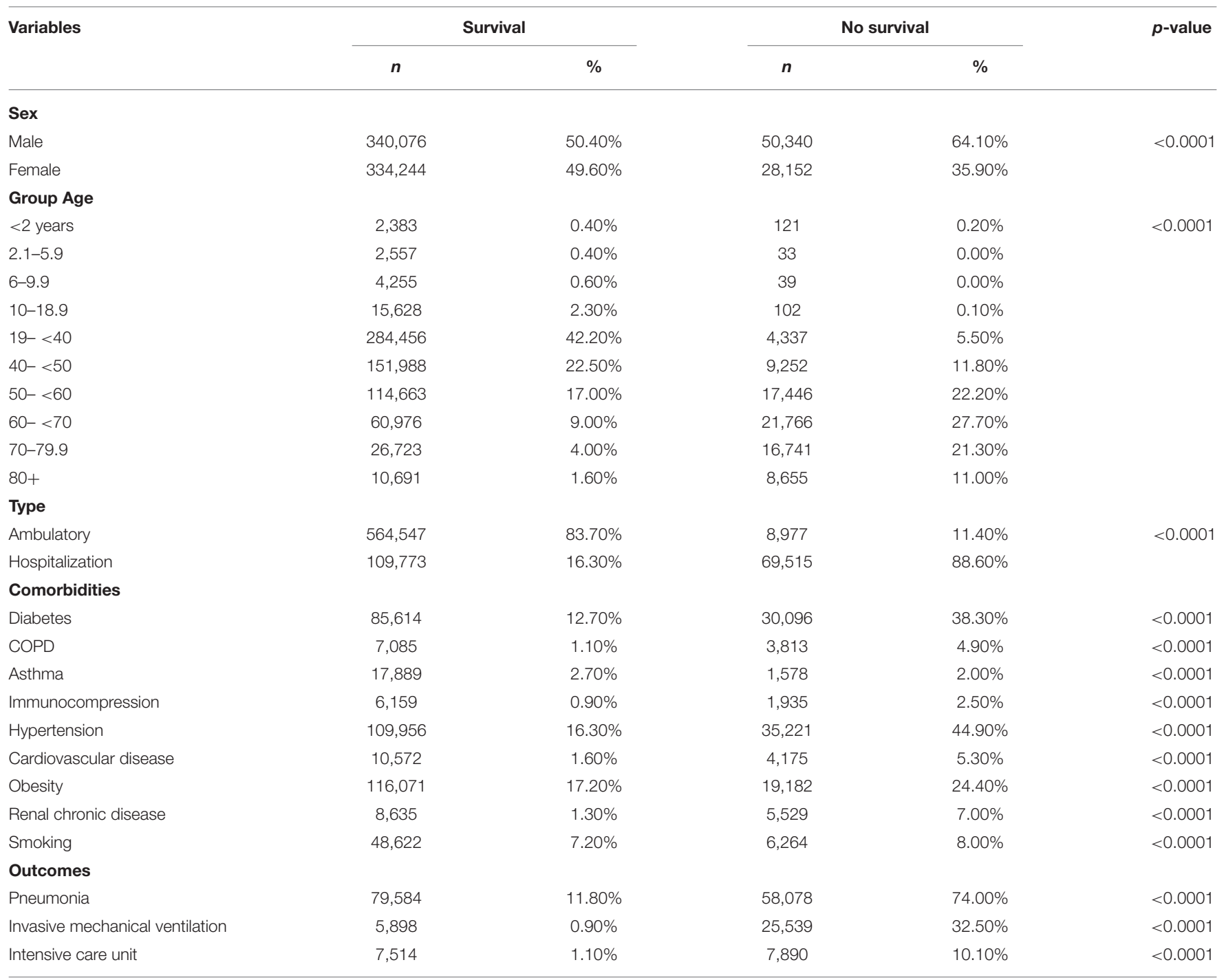

The value of $p$ was calculated by $X^{2}$ test. 